\title{
Synthesis of 2-Amino-4,6-diarylnicotinonitriles Using Silica-Bound $N$-Propyl Triethylenetetramine Sulfamic Acid as a Recyclable Solid Acid Catalyst
}

\author{
Khodabakhsh NIKNAM*, Abbas JAMALI, Marzieh TAJADDOD, Abdollah DERIS \\ Department of Chemistry, Faculty of Sciences, Persian Gulf University, Bushehr 75169, Iran
}

\begin{abstract}
A simple and efficient procedure for the preparation of silica-bound $N$-propyl triethylenetetramine sulfamic acid (SBPTETSA) by the reaction of silica-bound $N$-propyl triethylenetetramine (SBPTET) with chlorosulfonic acid in chloroform is described. Silica-bound $\mathrm{N}$-propyl triethylenetetramine sulfamic acid was employed as a recyclable catalyst for the synthesis of 2-amino-4,6-diarylnicotinonitriles from the multi-component reaction of an acetophenone derivative, an aromatic aldehyde, malononitrile, and ammonium acetate under solvent-free conditions at $100{ }^{\circ} \mathrm{C}$. The heterogeneous catalyst was recycled for five consecutive runs in the optimized multi-component reaction of 4-chloroacetophenone, 4-chloroenzaldehyde, malononitrile, and ammonium acetate without significant loses to its catalytic activity.
\end{abstract}

Key words: silica-bonded $N$-propyl triethylenetetramine sulfamic acid; 2-amino-4,6-diarylnicotinonitrile; pyridine; aldehyde; solvent-free CLC number: O643 Document code: A

Received 6 March 2012. Accepted 3 May 2012.

*Corresponding author. Tel: +98-771-4541494; Fax: +98-771-4545188; E-mail: khniknam@gmail.com

This work was supported by Persian Gulf University Research Council (PGU/FS/6-1/1390/705).

English edition available online at Elsevier ScienceDirect (http://www.sciencedirect.com/science/journal/18722067).

Pyridine and its derivatives are important structural motifs and are present in a great number of pharmaceutical compounds and natural products [1-6]. The unique structural arrays and highly pronounced biological and physiological activities [1-6] displayed by such compounds have made them attractive synthetic targets, with 2-amino-4,6diarylnicotinonitriles being especially considered as an important class of bio-active compounds [4-6]. For example, 2-aminopyridine derivatives have recently been identified as anti-microbial agents [7], IKK- $\beta$ inhibitors [8], and potent inhibitors of HIV-1 integrase [9]. Although there are multiple reports in the literature describing the synthesis of 2-amino-3-cyanopyridines, the majority of these procedures require multiple steps [10], long reaction times, toxic solvents such as benzene [11], high temperatures or microwave assistance [12], resulting in disappointingly low yields. In contrast, straight-forward and efficient one-pot catalytic procedures for the synthesis of 2-amino-3-cyanopyridines under mild conditions are still limited. Most recently, there have been reports describing the synthesis of 2-amino-3cyanopyridines according to a two step synthesis [13] using either a Brönsted acidic ionic liquid at $150{ }^{\circ} \mathrm{C}$ [14] or $\mathrm{Yb}(\mathrm{PFO})_{3}$ in refluxing ethanol [15].

Several types of solid sulfonic acid functionalized silica (both amorphous and ordered) have been synthesized and applied as alternatives to the traditional sulfonic acid resins and homogeneous acids in catalyzing chemical transforma- tions [16-24]. In a continuation of our own studies on the design and application of solid acid catalysts in organic transformations [17-24], we herein describe the preparation of silica-bound $N$-propyl triethylenetetramine sulfamic acid (SBPTETSA) and its application as a catalyst for the synthesis of 2-amino-4,6-diarylnicotininitriles.

SBPTETSA was prepared by the reaction of 3-triethylenetetramine- $N$-propylsilica (3-PNPS) with chlorosulfonic acid in chloroform (Scheme 1).

\section{Experimental}

\subsection{Catalyst preparation}

Chemicals were purchased from Fluka, Merck, and Aldrich. Fourier transform infrared (FT-IR) spectra were run on a Shimadzu Infrared spectroscopy FT-IR-8000 and a Bruker spectrum $\mathrm{X}$. The ${ }^{1} \mathrm{H}$ and ${ }^{13} \mathrm{C}$ NMR was run on Bruker Avance $(400 \mathrm{MHz})$ instruments in $\mathrm{CDCl}_{3}$. Melting points were recorded on a SMP1 melting point apparatus in open capillary tubes and are uncorrected. Reaction progress was followed by TLC using silica gel SILG/UV 254 plates. Chloropropyl silica was prepared according to a previously reported procedure [23]. All of the products were characterized by comparison of their IR, ${ }^{1} \mathrm{H}$, and ${ }^{13} \mathrm{C}$ NMR spectroscopic data and their melting points with the reported values [7-15]. 

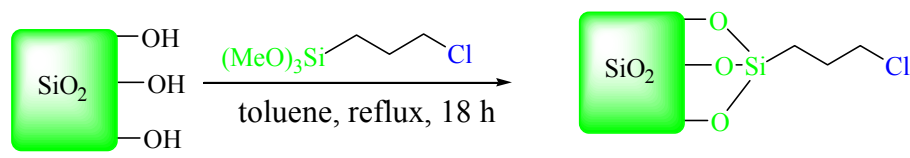

3-SPCl

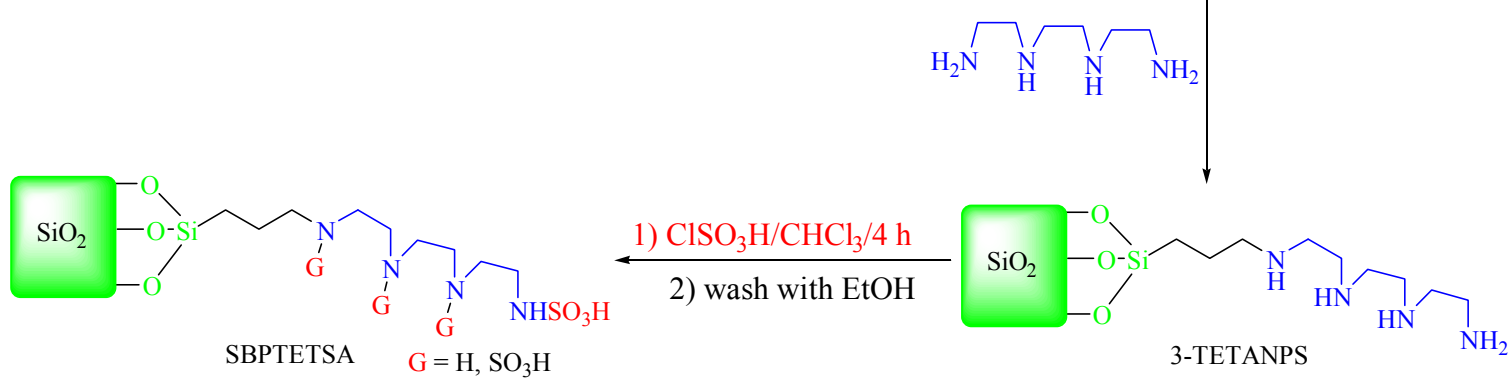

Scheme 1. Preparation of SBPTETSA.

\subsubsection{Synthesis of 3-triethylenetetramine- $N$ - propylsilica (3-TETANPS)}

To a mixture of chloropropyl silica (25 g) in anhydrous xylene $(250 \mathrm{ml})$, an excess of triethylenetetramine $(25 \mathrm{ml})$ was added. The resulting mixture was refluxed with stirring for $24 \mathrm{~h}$. The reaction mixture was then cooled to room temperature and filtered through a vacuum glass filter. The filter-cake was washed sequentially with xylene and a large excess of ethanol and dried under vacuum overnight at 80 ${ }^{\circ} \mathrm{C}$ to give the desired product 3-TETANPS (26.4 g).

\subsubsection{Synthesis of SBPTETSA}

To a magnetically stirred mixture of 3-TETANPS (26.4 g) in $\mathrm{CHCl}_{3}(50 \mathrm{ml})$ was added chlorosulfonic acid $(25 \mathrm{ml})$ in a drop-wise manner at $0{ }^{\circ} \mathrm{C}$ over a period of $2 \mathrm{~h}$. Upon completion of the reaction, the mixture was stirred for a further $2 \mathrm{~h}$ and then filtered. The filter-cake was washed with ethanol $(50 \mathrm{ml})$ and dried at room temperature to afford the desired product, SBPTETSA, as a cream powder $(27.5 \mathrm{~g})$.

Elemental analysis found: S, 1.04\%; C, 10.05\%; $\mathrm{H}$, $2.15 \%$; N, 2.54\%. The number of $\mathrm{H}^{+}$sites on the SBPTETSA was determined by $\mathrm{pH}$-ISE conductivity titration (Denver Instrument Model 270) and found to be 0.6 mmol of $\mathrm{H}^{+}$sites per $1 \mathrm{~g}$ of solid acid at $25^{\circ} \mathrm{C}(\mathrm{pH}=2.62)$.

\subsection{General procedure for the synthesis of 2-amino-4,6-diarylnicotininitrile derivatives}

To a mixture of an acetophenone derivative $(1.0 \mathrm{mmol})$, an aromatic aldehyde $(1.0 \mathrm{mmol})$, malononitrile $(1.0 \mathrm{mmol})$, and ammonium acetate $(1.5 \mathrm{mmol})$ was added SBPTETSA ( $0.07 \mathrm{~g}$, equivalent to $0.042 \mathrm{mmol}$ of $\mathrm{H}^{+}$) and the resulting mixture was heated at $100{ }^{\circ} \mathrm{C}$ under solvent-free conditions. Upon completion of the reaction (monitored by TLC), ethanol $(5 \mathrm{ml})$ was added and the resulting mixture was filtered. The filter-cake was washed with warm ethanol (5 $\mathrm{ml} \times 3$ ) to effectively clean the catalyst. The filtrates were subsequently combined and provided the products were recrystallized from ethanol. The recovered catalyst was dried and reused in the subsequent runs.

2-Amino-6-(4-chlorophenyl)-4-phenyl-nicotinonitrile (1a). IR (neat, $\mathrm{cm}^{-1}$ ): 3494.74, 3391.15, 2207.42, 1607.03, $1568.35,1546.79,1422.39,1364.52,1259.13,1084.75$, 1009.35, 829.34, 760.49, 693.35. ${ }^{1} \mathrm{H}$ NMR (400 MHz, $\left.\mathrm{CDCl}_{3}\right): \delta 5.29(\mathrm{~s}, 2 \mathrm{H}), 7.11(\mathrm{~s}, 1 \mathrm{H}), 7.38(\mathrm{~d}, 2 \mathrm{H}, J=8.8$ $\mathrm{Hz}), 7.45-7.47$ (m, 3H), 7.55-7.57 (m, 2H), 7.89 (d, 2H, $J=$ $8.8 \mathrm{~Hz}) .{ }^{13} \mathrm{C}$ NMR $\left(100 \mathrm{MHz}, \mathrm{CDCl}_{3}\right): \delta$ 109.95, 117.23, $127.12,127.58,127.96,127.99,131.77,135.40,139.69$, $154.73,157.42$.

2-Amino-4,6-bis-(4-chlorophenyl)-nicotinonitrile (1b). IR (neat, $\mathrm{cm}^{-1}$ ): 3498.89, 3393.13, 2204.81, 1607.13, $1579.10,1543.75,1492.07,1431.94,1396.80,1365.73$, 1258.38, 1091.86, 1011.82, 821.13, 773.54. ${ }^{1} \mathrm{H}$ NMR (400 $\left.\mathrm{MHz}, \mathrm{CDCl}_{3}\right): \delta 5.30(\mathrm{~s}, 2 \mathrm{H}), 7.07(\mathrm{~s}, 1 \mathrm{H}), 7.38(\mathrm{~d}, 2 \mathrm{H}, J=$ $8.0 \mathrm{~Hz}), 7.44(\mathrm{~d}, 2 \mathrm{H}, J=8.0 \mathrm{~Hz}), 7.50(\mathrm{~d}, 2 \mathrm{H}, J=8.0 \mathrm{~Hz})$, $7.88(\mathrm{~d}, 2 \mathrm{H}, J=8.0 \mathrm{~Hz}) .{ }^{13} \mathrm{C} \mathrm{NMR}\left(100 \mathrm{MHz}, \mathrm{CDCl}_{3}\right): \delta$ $110.68,116.79,122.17,128.62,129.07,129.31,129.51$, $154.99,160.32$.

2-Amino-6-(4-chlorophenyl)-4-(4-methoxyphenyl)-nicoti nonitrile (1c). IR (neat, $\mathrm{cm}^{-1}$ ): 3458.62, 3365.89, 2917.65, $2206.41,1582.36,1544.06,1515.45,1454.96,1427.14$, 1367.80, 1297.85, 1254.62, 1183.98, 1012.28, 821.40. ${ }^{1} \mathrm{H}$ NMR (400 MHz, $\mathrm{CDCl}_{3}$ ): $\delta 3.82(\mathrm{~s}, 3 \mathrm{H}), 5.25(\mathrm{~s}, 2 \mathrm{H}), 6.97$ (d, 2H, $J=8.0 \mathrm{~Hz}), 7.09$ (s, 1H), 7.37 (d, 2H, $J=8.0 \mathrm{~Hz})$, 7.54 (d, 2H, $J=8.0 \mathrm{~Hz}), 7.88(\mathrm{~d}, 2 \mathrm{H}, J=8.0 \mathrm{~Hz}) .{ }^{13} \mathrm{C} \mathrm{NMR}$ $\left(100 \mathrm{MHz}, \mathrm{CDCl}_{3}\right): \delta$ 65.36, 109.71, 127.55, 127.96, $128.59,132.37,135.29,138.39,154.36,159.78$.

2-Amino-6-(4-chlorophenyl)-4-(4-methylsulfanylphenyl) -nicotinonitrile (1d). IR (neat, $\mathrm{cm}^{-1}$ ): 3494.70, 3392.31, 2919.98, 2206.92, 1608.73, 1572.57, 1539.05, 1493.20, 
1435.71, 1364.39, 1259.07, 1087.49, 1011.87, 806.21. ${ }^{1} \mathrm{H}$ NMR $\left(400 \mathrm{MHz}, \mathrm{CDCl}_{3}\right): \delta 2.47(\mathrm{~s}, 3 \mathrm{H}), 5.28(\mathrm{~s}, 2 \mathrm{H}), 7.08$ (s, $1 \mathrm{H}), 7.30(\mathrm{~d}, 2 \mathrm{H}, J=8.0 \mathrm{~Hz}), 7.37(\mathrm{~d}, 2 \mathrm{H}, J=8.0 \mathrm{~Hz})$, 7.49 (d, $2 \mathrm{H}, J=8.0 \mathrm{~Hz}), 7.88(\mathrm{~d}, 2 \mathrm{H}, J=8.0 \mathrm{~Hz}) .{ }^{13} \mathrm{C} \mathrm{NMR}$ $\left(100 \mathrm{MHz}, \mathrm{CDCl}_{3}\right): \delta 14.22,109.63,125.15,127.45$, $127.56,127.99,131.94,135.26,140.63,153.62,157.43$, 159.20. Anal calcd for $\mathrm{C}_{19} \mathrm{H}_{14} \mathrm{ClN}_{3} \mathrm{~S}: \mathrm{C}, 64.86 ; \mathrm{H}, 4.01 ; \mathrm{N}$, 11.94; Found: C, 64.67; H, 4.10; N, 11.78.

2-Amino-6-(4-chlorophenyl)-4-p-tolyl-nicotinonitrile (1e). IR (neat, $\mathrm{cm}^{-1}$ ): 3493.41, 3389.76, 2919.01, 2204.78, $1606.55,1578.89,1543.48,1366.12,1257.48,1048.54$, 811.25. ${ }^{1} \mathrm{H}$ NMR (400 MHz, $\left.\mathrm{CDCl}_{3}\right): \delta 2.37(\mathrm{~s}, 3 \mathrm{H}), 5.27$ (s, $2 \mathrm{H}), 7.09(\mathrm{~s}, 1 \mathrm{H}), 7.26(\mathrm{~d}, 2 \mathrm{H}, J=7.8 \mathrm{~Hz}), 7.37$ (d, 2H, $J=$ $8.8 \mathrm{~Hz}), 7.46(\mathrm{~d}, 2 \mathrm{H}, J=10.1 \mathrm{~Hz}), 7.88(\mathrm{~d}, 2 \mathrm{H}, J=8.8 \mathrm{~Hz})$. ${ }^{13} \mathrm{C}$ NMR $\left(100 \mathrm{MHz}, \mathrm{CDCl}_{3}\right): \delta$ 20.36, 109.84, 127.01, $127.55,127.96,128.65,132.84,135.31,139.19,154.31$, 159.15. Anal calcd for $\mathrm{C}_{19} \mathrm{H}_{14} \mathrm{ClN}_{3}$ : C, 71.36; $\mathrm{H}, 4.41 ; \mathrm{N}$, 11.09; Found: C, 71.18; H, 4.52; N, 10.94.

2-Amino-6-(4-fluorophenyl)-4-p-tolyl-nicotinonitrile (1f). IR (neat, $\mathrm{cm}^{-1}$ ): 3491.85, 3391.49, 2908.20, 2206.03, $1606.46,1581.58,1546.75,1508.21,1427.95,1368.11$, $1302.75, \quad 1259.52,1217.55,1158.98,837.04,817.49$, 773.16. ${ }^{1} \mathrm{H}$ NMR $\left(400 \mathrm{MHz}, \mathrm{CDCl}_{3}\right): \delta 2.37(\mathrm{~s}, 3 \mathrm{H}), 5.26(\mathrm{~s}$, $2 \mathrm{H}), 7.06-7.10(\mathrm{~m}, 3 \mathrm{H}), 7.26(\mathrm{~d}, 2 \mathrm{H}, J=7.8 \mathrm{~Hz}), 7.46(\mathrm{dt}$, $\left.2 \mathrm{H}, J_{1}=8.0 \mathrm{~Hz}, J_{2}=2.0 \mathrm{~Hz}\right), 7.91-7.95(\mathrm{~m}, 2 \mathrm{H}) .{ }^{13} \mathrm{C} \mathrm{NMR}$ $\left(100 \mathrm{MHz}, \mathrm{CDCl}_{3}\right): \delta 21.41,110.80,115.70,115.91$, $128.06,129.26,129.34,129.69,133.94,140.19,160.19$. Anal calcd for $\mathrm{C}_{19} \mathrm{H}_{14} \mathrm{FN}_{3}: \mathrm{C}, 75.23 ; \mathrm{H}, 4.65 ; \mathrm{N}, 13.85$; Found: C, 75.04; H, 4.58; N, 13.78.

2-Amino-6-(4-fluorophenyl)-4-(4-methoxyphenyl)-nicoti nonitrile (1g). IR (neat, $\mathrm{cm}^{-1}$ ): 3449.08, 3322.16, 2906.23, $2210.69,1600.97,1573.26,1546.15,1511.12,1441.74$, $1372.80,1296.30,1255.32,1226.99,1177.52,1027.28$, 857.28, 838.65, 817.59. ${ }^{1} \mathrm{H}$ NMR ( $\left.400 \mathrm{MHz}, \mathrm{CDCl}_{3}\right): \delta 3.81$ $(\mathrm{s}, 3 \mathrm{H}), 5.25(\mathrm{~s}, 2 \mathrm{H}), 6.97\left(\mathrm{dt}, 2 \mathrm{H}, J_{1}=8.8 \mathrm{~Hz}, J_{2}=2.5 \mathrm{~Hz}\right)$, $7.06-7.11(\mathrm{~m}, 3 \mathrm{H}), 7.53\left(\mathrm{dt}, 2 \mathrm{H}, J_{1}=9.1 \mathrm{~Hz}, J_{2}=2.7 \mathrm{~Hz}\right)$, $7.90-7.95(\mathrm{~m}, 2 \mathrm{H}) .{ }^{13} \mathrm{C}$ NMR $\left(100 \mathrm{MHz}, \mathrm{CDCl}_{3}\right): \delta 55.46$, $110.65,114.40,115.69,115.90,129.25,129.33,129.62$, $154.85,158.51,160.64\left(J_{\text {C-F }}=194.73\right)$. Anal calcd for $\mathrm{C}_{19} \mathrm{H}_{14} \mathrm{FN}_{3} \mathrm{O}$ : C, 71.46; H, 4.42; N, 13.16; Found: C, 71.28; $\mathrm{H}, 4.40 ; \mathrm{N}, 13.02$.

2-Amino-6-(4-fluorophenyl)-4-(4-chlorophenyl)-nicotino nitrile (1h). IR (neat, $\mathrm{cm}^{-1}$ ): 3480.19, 3346.41, 2217.26, $1632.44,1596.41,1544.46,1510.16,1491.50,1441.34$, $1362.47,1226.12,1152.51,1086.58,1011.67,808.05 .{ }^{1} \mathrm{H}$ NMR (400 MHz, $\left.\mathrm{CDCl}_{3}\right): \delta 5.30(\mathrm{~s}, 2 \mathrm{H}), 7.05(\mathrm{~s}, 1 \mathrm{H}), 7.09$ (t, $2 \mathrm{H}, J=8.7 \mathrm{~Hz}), 7.44$ (d, 2H, $J=8.3 \mathrm{~Hz}), 7.50(\mathrm{~d}, 2 \mathrm{H}, J=$ $8.6 \mathrm{~Hz}), 7.92-7.95(\mathrm{~m}, 2 \mathrm{H}) .{ }^{13} \mathrm{C}$ NMR $\left(100 \mathrm{MHz}, \mathrm{CDCl}_{3}\right): \delta$ $110.60,115.78,115.99,116.87,129.30,129.39,129.51$, $133.87,135.21,136.22,153.98,159.52\left(J_{\mathrm{C}-\mathrm{F}}=129.21 \mathrm{~Hz}\right)$. Anal. Calcd for $\mathrm{C}_{18} \mathrm{H}_{11} \mathrm{ClFN}_{3}$ : C, 66.78; H, 3.42; N, 12.98; Found: C, 66.62; H, 3.37; N, 12.81.
2-Amino-6-(4-fluorophenyl)-4-(2-chlorophenyl)-nicotino nitrile (1i). IR (neat, $\mathrm{cm}^{-1}$ ): 3485.17, 3347.14, 2223.97, $1626.98,1596.59,1551.96,1508.63,1439.86,1361.06$, $1223.80,1156.26,1054.21,852.13,826.12,759.32 .{ }^{1} \mathrm{H}$ NMR (400 MHz, $\left.\mathrm{CDCl}_{3}\right): \delta 5.28(\mathrm{~s}, 2 \mathrm{H}), 7.03(\mathrm{~s}, 1 \mathrm{H}), 7.08$ (t, $2 \mathrm{H}, J=8.7 \mathrm{~Hz}), 7.29-7.38(\mathrm{~m}, 3 \mathrm{H}), 7.47-7.49(\mathrm{~m}, 1 \mathrm{H})$, 7.91-7.96 (m, 2H). ${ }^{13} \mathrm{C}$ NMR (100 MHz, $\left.\mathrm{CDCl}_{3}\right): \delta 111.83$, $115.74,115.95,116.19,127.15,129.45,130.28,130.32$, $130.75,132.27,133.88,135.77,153.13,158.48,159.57$, $164.12\left(J_{\mathrm{C}-\mathrm{F}}=251.03 \mathrm{~Hz}\right)$. Anal. Calcd for $\mathrm{C}_{18} \mathrm{H}_{11} \mathrm{ClFN}_{3}: \mathrm{C}$, 66.78; H, 3.42; N, 12.98; Found: C, 66.59; H, 3.39; N, 12.75 .

2-Amino-4-phenyl-6- $p$-tolyl-nicotinonitrile (1j). IR (neat, $\left.\mathrm{cm}^{-1}\right): 3491.20, \quad 3392.15, \quad 2918.62,2204.33,1627.25$, $1574.03,1542.94,1425.23,1369.13,1257.57,1018.03$, $818.58,767.55,704.04 .{ }^{1} \mathrm{H}$ NMR $\left(400 \mathrm{MHz}, \mathrm{CDCl}_{3}\right): \delta 2.35$ (s, 3H), $5.31(\mathrm{~s}, 2 \mathrm{H}), 7.11(\mathrm{~s}, 1 \mathrm{H}), 7.25(\mathrm{~d}, 2 \mathrm{H}, J=8.0 \mathrm{~Hz})$, 7.37-7.41 (m, 3H), $7.46(\mathrm{~d}, 2 \mathrm{H}, J=8.0 \mathrm{~Hz}), 7.90-7.92(\mathrm{~m}$, $2 \mathrm{H}) .{ }^{13} \mathrm{C}$ NMR $\left(100 \mathrm{MHz}, \mathrm{CDCl}_{3}\right): \delta 20.34,110.13,116.31$, $126.28,127.04,127.77,128.61,129.11,132.99,136.99$, $139.06,154.11,158.71,159.26$.

2-Amino-4-(4-nitrophenyl)-6-p-tolyl-nicotinonitrile (1k). IR (neat, $\mathrm{cm}^{-1}$ ): 3491.25, 3388.15, 2920.08, 2206.90, $1606.18,1549.34,1512.86,1349.58,1258.08,1012.06$, 791.23, 699.25. ${ }^{1} \mathrm{H}$ NMR $\left(400 \mathrm{MHz}, \mathrm{CDCl}_{3}\right): \delta 2.35(\mathrm{~s}, 3 \mathrm{H})$, $5.34(\mathrm{~s}, 2 \mathrm{H}), 7.11(\mathrm{~s}, 1 \mathrm{H}), 7.23(\mathrm{~d}, 2 \mathrm{H}, J=8.0 \mathrm{~Hz}), 7.73(\mathrm{dd}$, $\left.2 \mathrm{H}, J_{1}=9.1 \mathrm{~Hz}, J_{2}=2.2 \mathrm{~Hz}\right), 7.84\left(\mathrm{dd}, 2 \mathrm{H}, J_{1}=8.3 \mathrm{~Hz}, J_{2}=\right.$ $1.8 \mathrm{~Hz}), 8.32\left(\mathrm{dd}, 2 \mathrm{H}, J_{1}=9.1 \mathrm{~Hz}, J_{2}=2.0 \mathrm{~Hz}\right) .{ }^{13} \mathrm{C} \mathrm{NMR}$ $\left(100 \mathrm{MHz}, \mathrm{CDCl}_{3}\right): \delta 28.67,123.15,126.25,128.33$, 128.69, 129.21, 164.45. Anal calcd for $\mathrm{C}_{19} \mathrm{H}_{14} \mathrm{~N}_{4} \mathrm{O}_{2}$ : C, 69.08; H, 4.27; N, 16.96; Found: C, 68.91; H, 4.19; N, 16.78 .

2-Amino-4-(3-nitrophenyl)-6-p-tolyl-nicotinonitrile (11). IR (neat, $\mathrm{cm}^{-1}$ ): 3479.98, 3365.81, 2217.73, 1622.34, $1568.24,1525.06,1342.43,1258.22,1084.85,813.11$, 743.43. ${ }^{1} \mathrm{H}$ NMR (400 MHz, $\left.\mathrm{CDCl}_{3}\right): \delta 2.35(\mathrm{~s}, 3 \mathrm{H}), 5.34(\mathrm{~s}$, 2H), $7.13(\mathrm{~s}, 1 \mathrm{H}), 7.23$ (d, 2H, $J=7.8 \mathrm{~Hz}), 7.67$ (t, $1 \mathrm{H}, J=$ $8.1 \mathrm{~Hz}), 7.86(\mathrm{~d}, 2 \mathrm{H}, J=8.3 \mathrm{~Hz}), 7.92(\mathrm{~d}, 1 \mathrm{H}, J=8.1 \mathrm{~Hz})$, 8.30-8.32 (m, 1H), 8.40-8.41 (m, 1H). ${ }^{13} \mathrm{C}$ NMR $(100$ $\left.\mathrm{MHz}, \mathrm{CDCl}_{3}\right): \delta 21.46,110.61,123.33,124.46,127.32$, 129.67, 130.12, 134.19, 138.62, 141.10, 152.28, 160.20 . Anal calcd for $\mathrm{C}_{19} \mathrm{H}_{14} \mathrm{~N}_{4} \mathrm{O}_{2}$ : C, 69.08; $\mathrm{H}, 4.27 ; \mathrm{N}, 16.96$; Found: C, 68.89; H, 4.14; N, 16.73.

\section{Results and discussion}

The thermogravimetric analysis (TGA) curve of SBPTETSA revealed the mass loss of organic materials as they decomposed upon heating (Fig. 1). An initial mass loss from the SBPTETSA up to $120{ }^{\circ} \mathrm{C}$ was caused by the removal of physically adsorbed solvent and surface hydroxyl groups. A mass loss accounting for approximately $11.58 \%$ 


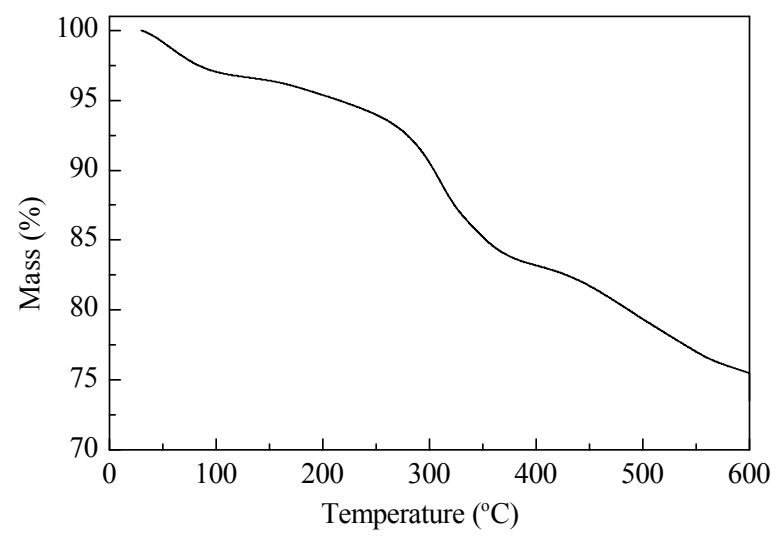

Fig. 1. TGA profile of SBTETASA.

of the initial mass occurred between 220 and $410{ }^{\circ} \mathrm{C}$ and was attributed to the thermal decomposition of the organic groups. The covalent chemical bonds within the catalyst bestowed high thermal stability on the structure.

The FT-IR spectrum of SBPTETSA is shown in Fig. 2. The major peaks for silica $\left(\mathrm{SiO}_{2}\right)$ are the broad anti-symmetric Si-O-Si stretching mode from 1300 to 1000 $\mathrm{cm}^{-1}$ and the symmetric $\mathrm{Si}-\mathrm{O}-\mathrm{Si}$ stretching mode near $820-750 \mathrm{~cm}^{-1}$. For sulfamic acid functional group, the FT-IR absorption ranges of the $\mathrm{O}=\mathrm{S}=\mathrm{O}$ asymmetric and symmetric stretching modes were located at 1170 and 1060 $\mathrm{cm}^{-1}$, respectively. The FT-IR spectrum revealed an overlap in the asymmetric and symmetric stretching bands of $\mathrm{SO}_{2}$ with the $\mathrm{Si}-\mathrm{O}-\mathrm{Si}$ stretching bands in the silica functionalized alkyl-sulfamic acid. The spectrum also contained a broad $\mathrm{OH}$ stretching absorption from 3600 to $3200 \mathrm{~cm}^{-1}$. $\mathrm{NH}$ stretching and bending occurred at 3420 and $1650 \mathrm{~cm}^{-1}$, respectively.

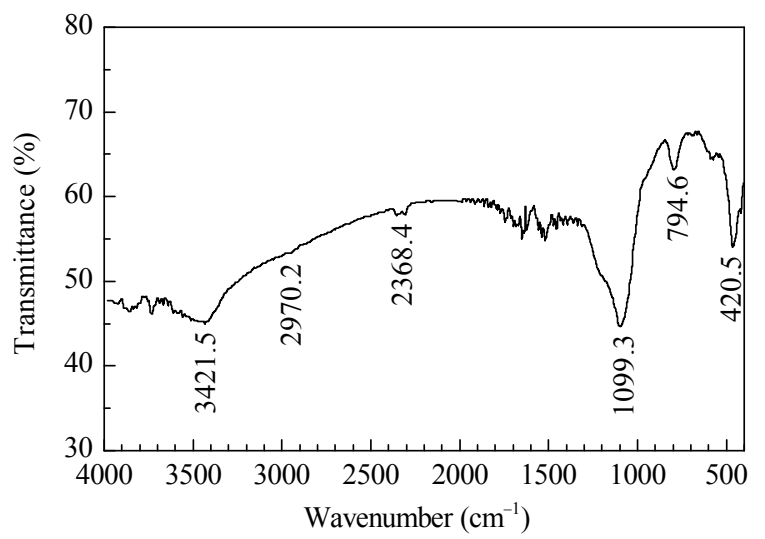

Fig. 2. FT-IR spectrum of SBTETASA.

The one-pot synthesis of $\mathbf{1 b}$ was selected as a model reaction for our study. A mixture of 4-chlorobenzaldehyde (1 mmol), 4-chloroacetophenone (1 mmol), malononitrile (1 $\mathrm{mmol})$, ammonium acetate $(1.5 \mathrm{mmol})$, and different catalytic loading of SBPTETSA was stirred under solvent-free conditions at $100{ }^{\circ} \mathrm{C}$ (Table 1). It is clear from the results that SBPTETSA performed as an effective catalyst in this four-component condensation reaction. The best catalytic loading of SBPTETSA from the experiments conducted was $0.07 \mathrm{~g}$ in terms of reaction time and isolated yield. The application of a lower temperature gave the corresponding product in lower yield, with an extended reaction time being required to drive the reaction to higher levels of conversion (Table 1, entry 6). The reaction conditions outlined in entry 3 of Table 1 were subsequently identified as the optimized conditions, involving the heating of a mixture of an acetophenone derivative (1 mmol), an aldehyde (1 $\mathrm{mmol})$, malononitrile (1 mmol), ammonium acetate $(1.5 \mathrm{mmol})$, and SBPTETSA $\left(0.07 \mathrm{~g}\right.$, equal to $0.04 \mathrm{mmol}$ of $\left.\mathrm{H}^{+}\right)$at $100{ }^{\circ} \mathrm{C}$ under solvent-free conditions.

Table 1 Condensation reaction of 4-chloroacetophenone, 4-chlorobenzaldehyde, malononitrile, and ammonium acetate in the presence of different loading of the catalyst under solvent-free conditions at different temperatures

\begin{tabular}{ccccc}
\hline Entry & Catalyst & $\begin{array}{c}\text { Catalyst } \\
\text { loading }(\mathrm{g})\end{array}$ & $\begin{array}{c}\text { Temperature } \\
\left({ }^{\circ} \mathrm{C}\right)\end{array}$ & $\begin{array}{c}\text { Yield }^{*} \\
(\%)\end{array}$ \\
\hline 1 & SBTETASA & 0.01 & 100 & 65 \\
2 & SBTETASA & 0.03 & 100 & 75 \\
3 & SBTETASA & 0.07 & 100 & 90 \\
4 & SBTETASA & 0.1 & 100 & 91 \\
5 & SBTETASA & 0.07 & 120 & 90 \\
6 & SBTETASA & 0.07 & 90 & 80 \\
7 & $\mathrm{H}_{2} \mathrm{SO}_{4}$ & $0.1(1 \mathrm{mmol})$ & 100 & trace \\
8 & $\mathrm{CH}_{3} \mathrm{COOH}$ & $0.1(1.67 \mathrm{mmol})$ & 100 & 40 \\
9 & $\mathrm{H}_{2} \mathrm{NSO}_{3} \mathrm{H}$ & $0.07(0.72 \mathrm{mmol})$ & 100 & 70 \\
\hline
\end{tabular}

Reaction conditions: 4-chlorobenzaldehyde $1 \mathrm{mmol}$, 4-chloroacetophenone $1 \mathrm{mmol}$, malononitrile $1 \mathrm{mmol}$, ammonium acetate $1.5 \mathrm{mmol}$, $10 \mathrm{~min}$. *Isolated yield

With the optimized reaction conditions in hand, we proceeded to investigate the scope of the reaction by employing a wide range of aromatic aldehydes in the process (Table 2). The reaction performed well, providing the corresponding 2-amino-3-cyanopyridines in good to high yields, demonstrating the generality of the method and its good tolerance of both electron-withdrawing and electron-donating substituents on the aromatic ring of the aldehyde. For example, aromatic aldehydes containing electron-donating substituents, including 4-OMe, 4-SMe, and 4-Me, reacted with acetophenone derivatives, including 4-chloro- and 4-fluoroacetophenone under the optimized conditions, to provide the corresponding products in good to high yields (Table 2, entries 3-7).

Furthermore, aromatic aldehydes containing electron-withdrawing substituents, including $4-\mathrm{NO}_{2}$ and $3-\mathrm{NO}_{2}$ reacted with 4-methyl-acetophenone and malononitrile under the optimized conditions to afford the corresponding 
Table 2 Synthesis of various 2-amino-4,6-diarylnicotinonitriles in the presence of SBPTETSA under solvent-free conditions

\begin{tabular}{|c|c|c|c|c|c|}
\hline & & & & \multicolumn{2}{|r|}{$1 \mathrm{a}-11$} \\
\hline Entry & $\mathrm{R}^{1}$ & $\mathrm{R}^{2}$ & Product & Yield $^{*}(\%)$ & Melting point $\left({ }^{\circ} \mathrm{C}\right)$ \\
\hline 1 & $\mathrm{H}$ & $4-\mathrm{Cl}$ & $1 \mathbf{a}$ & 85 & $240-242(240-241$ [13]) \\
\hline 2 & $4-\mathrm{Cl}$ & $4-\mathrm{Cl}$ & $1 \mathrm{~b}$ & 90 & $230-231(228-229[13])$ \\
\hline 3 & $4-\mathrm{MeO}$ & $4-\mathrm{Cl}$ & $1 \mathrm{c}$ & 75 & $204-205$ \\
\hline 4 & 4-MeS & $4-\mathrm{Cl}$ & 1d & 65 & $202-203$ \\
\hline 5 & $4-\mathrm{Me}$ & $4-\mathrm{Cl}$ & $1 e$ & 85 & $215-217$ \\
\hline 6 & $4-\mathrm{Me}$ & $4-\mathrm{F}$ & 1f & 80 & $203-204$ \\
\hline 7 & $4-\mathrm{MeO}$ & $4-\mathrm{F}$ & $1 \mathrm{~g}$ & 85 & 194-196 \\
\hline 8 & $4-\mathrm{Cl}$ & $4-\mathrm{F}$ & $1 \mathrm{~h}$ & 90 & $204-206$ \\
\hline 9 & $2-\mathrm{Cl}$ & $4-\mathrm{F}$ & $1 \mathbf{i}$ & 87 & $202-203$ \\
\hline 10 & $\mathrm{H}$ & 4-Me & $1 \mathbf{j}$ & 80 & $182-183$ \\
\hline 11 & $4-\mathrm{O}_{2} \mathrm{~N}$ & $4-\mathrm{Me}$ & $1 \mathrm{k}$ & 65 & $201-203(180-182$ [13]) \\
\hline 12 & $3-\mathrm{O}_{2} \mathrm{~N}$ & 4-Me & 11 & 67 & $188-190$ \\
\hline
\end{tabular}

Reaction conditions: aldehyde $1 \mathrm{mmol}$, acetophenone derivative $1 \mathrm{mmol}$, malononitrile $1 \mathrm{mmol}$, ammonium acetate $1.5 \mathrm{mmol}$, SBTETASA $0.07 \mathrm{~g}$ (equal to $0.042 \mathrm{mmol}$ of $\mathrm{H}^{+}$), $100{ }^{\circ} \mathrm{C}, 10 \mathrm{~min}$. ${ }^{*}$ Isolated yield.

products in high yield (Table 2, entries 11 and 12). Ortho-substituted aromatic aldehydes also reacted successfully in this four component condensation (Table 2, entry 9). It is worthy of note that this method not only affords the products in good to high yields but also effectively avoids problems associated with catalyst cost, handling, safety, and pollution.

The possibility of recycling the catalyst was examined using the reaction of 4-chlorobenzaldehyde, 4-chloroacetophenone, malononitrile, and ammonium acetate under the optimized conditions (Fig. 3). Upon completion of the reaction, the mixture was filtered and the filter-cake was washed

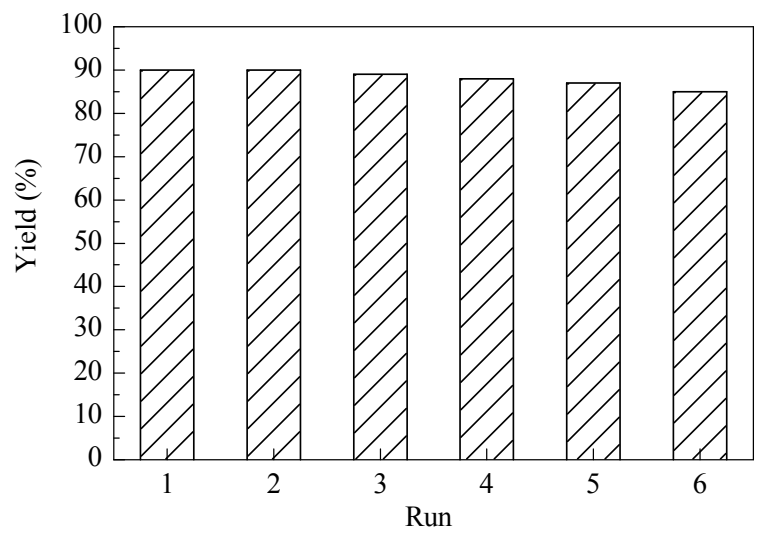

Fig. 3. Recyclability of the SBPTETSA catalyst. Reaction conditions: 4-chloroacetophenone $1 \mathrm{mmol}$, 4-chlorobenzaldehyde $1 \mathrm{mmol}$, malononitrile $1 \mathrm{mmol}$, ammonium acetate $1.5 \mathrm{mmol}$, catalyst $0.07 \mathrm{~g}$, $100{ }^{\circ} \mathrm{C}, 10 \mathrm{~min}$. with ethanol. The filter-cake of solid catalyst was then collected and stored ahead of the next reaction. The recycled catalyst could be reused five times without any further treatment. Furthermore, no appreciable loss was observed in the catalytic activity of the SBPTETSA catalysts over the five recycling runs.

\section{Conclusions}

Silica-bound propyl triethylenetetramine sulfamic acid efficiently catalyzed the synthesis of 2-amino-4,6-diarylnicotinonitriles via a four component condensation reaction. The simplicity of this procedure, together with the eco-friendly, non-volatile, easy-to-handle, non-hazardous, and recyclable nature of catalyst, represents the main advantages of this method.

\section{Acknowledgments}

We are thankful to the School of Chemistry, Manchester University for running NMR, CHN, and TGA.

\section{References}

1 Cocco M T, Congiu C, Lilliu V, Onnis V. Eur J Med Chem, 2005, 40: 1365

2 Fredholm B B, Ijzerman A P, Jacobson K A, Klotz K N, Linden J. Pharmacol Rev, 2001, 53: 527

3 Guo K, Thompson M J, Chen B. J Org Chem, 2009, 74: 6999 
4 May B C H, Zorn J A, Witkop J, Sherrill J, Wallace A C, Legname G, Prudiner S B, Cohen F E. J Med Chem, 2007, 50: 65

5 Guo K, Mutter R, Heal W, Reddy T R K, Cope H, Pratt S, Thompson M J, Chen B. Eur J Med Chem, 2008, 43: 93

6 Cocco M T, Congiu C, Lilliu V, Onnis V. Bioorg Med Chem, 2007, 15: 1859

7 Khidre R E, Abu-Hashem A A, El-Shazly M. Eur J Med Chem, 2011, 46: 5057

8 Murata T, Shimada M, Sakakibara S, Yoshinno T, Kadono H, Masuda T, Shimazaki M, Shintani T, Fuchikami K, Saki K, Inbe H, Takeshita K, Niki T, Umeda M, Bacon K B, Ziegelbauer K B, Lowinger T B. Bioorg Med Chem Lett, 2003, 13: 913

9 Mantri M, De Graaf O, Van Valdhoven J, Gobluos A, Von Frijtag Drabbe Kunzel J K, Mulder-Krieger T, Link R, De Vries H, Beukers M W, Brussee J, Ijzerman A P. J Med Chem, 2008, 51: 4449

10 Girgis A S, Kalmouch A, Hosni H M. Amino Acids, 2004, 26: 139

11 Kambe S, Saito K, Sakurai A, Midorikawa H. Synthesis, 1980: 366
12 Shi F, Tu S J, Fang F, Li T J. Arkivoc, 2005, (1): 137

13 Shah H C, Shah V H, Desai N D. Arkivoc, 2009, (2): 76

14 Davoodnia A, Attar P, Morsali A, Eshghi H, Tavakoli-Hoseini N, Khadem S. Bull Korean Chem Soc, 2011, 32: 1873

15 Tang J, Wang L M, Yao Y F, Zhang L, Wang W B. Tetrahedron Lett, 2011, 52: 509

16 Zareyee D, Karimi B. Tetrahedron Lett, 2007, 48: 1277

17 Niknam K, Saberi D, Nouri Sefat M. Tetrahedron Lett, 2009, 50: 4058

18 Niknam K, Saberi D. Tetrahedron Lett, 2009, 50: 5210

19 Niknam K, Saberi D. Appl Catal A, 2009, 366: 220

20 Niknam K, Saberi D, Sadegheyan M, Deris A. Tetrahedron Lett, 2010, 51: 692

21 Niknam K, Saberi D, Nouri Sefat M. Tetrahedron Lett, 2010, 51: 2959

22 Niknam K, Deris A, Naeimi F, Majleci F. Tetrahedron Lett, 2011, 52: 4642

23 Nouri Sefat M, Saberi D, Niknam K. Catal Lett, 2011, 141: 1713

24 Tayebi S, Baghernejad M, Saberi D, Niknam K. Chin J Catal, 2011, 32: 1477 